\title{
Research on Digital Transformation of Securities Companies Enabled by Financial Technology
}

\author{
Huimin $\mathrm{Li}^{*}$
}

\author{
Zhongtai Securities Co.,Ltd, Jinan, China \\ ${ }^{*}$ Corresponding author. Email: lihuimin9377@126.com
}

\begin{abstract}
In this paper, by constructing the security data management company and innovation laboratory, we not only systematically grasp the feature of the industry chain value model, and the entity industry asset securitization and its flow pattern, supervision, and risk prevention, but also provide a comprehensive study of the development, integration, and application of the related techniques that are processed, quantitative, indexing, measurable, and modeling. Accordingly, we carry out all-around innovative research and design of the value analysis model of the whole industry chain to realize the digital transformation of securities companies enabled by financial technology. On this basis, this paper also proposes ideas of discovering and integrating core data sources of the securities companies and constructing industry (enterprise) Knowledge Graph database, value chain model, big data risk management service platform, and securities big data intelligent service expert system from the perspective of the customer, product, business, respectively. Finally, we conduct a general discussion of the application and business embedding of artificial intelligence technology.
\end{abstract}

Keywords: Financial technology, Enabled, Digital transformation of securities companies.

\section{金融科技赋能证券公司数字化转型研究}

\author{
李会民*
}

中泰证券股份有限公司, 济南, 中国

*通讯作者. 邮箱: lihuimin9377@126.com

中文摘要

本文通过构建证券公司数据管理公司和创新实验室, 系统把握证券行业产业价值链模型、实体产业资产证券化 及其流动模式、监管与风险防控模式等需要的流程化、定量化、指标化、测量性、模型化等相关的技术开发整 合、集成与应用, 开展全方位全产业链价值分析模式的创新研究与设计, 实现金融科技赋能数字化转型。在此 基础上, 文章还提出了针对证券公司核心数据源数据进行发现和集成, 从客户、产品、业务等视角构建证券行 业知识图谱库和价值链模型、大数据风险管理服务平台、证券大数据智能化服务专家系统的设想。最后, 文章 还对人工智能技术的应用和业务嵌入进行了探讨。

关键词：金融科技，赋能，证券公司数字化转型 


\section{1. 引言}

2020 年新冠疫情加速了企业数字化转型的步伐, 在疫情倒逼下, 数字化转型已经由虚入实, 从概念探 索到规模化落地阶段, 许多企业开始构建数字化发展 的新生态。数字化转型没有适用于所有企业的统一的 模式可借鉴，如何立足于顶端设计，结合自身特点， 以及未来的产业互联、行业生态, 开发数字化技术和 支持能力, 完成数字化商业模式的创新与重构, 是证 券公司在推进数字化转型进程中需要面临的新课题。

\section{2. 证券行业金融科技与应用现状}

目前, 国家金融开放已呈加速趋势, 国际化要素 的融入越来越广泛, 科技生态、风险生态和行业发展 环境交织在一起, 金融科技领域的监管法规、技术架 构、行业应用也已逐步形成相对完善的发展框架。受 其影响, 证券公司急需加大底层基础设施建设, 叠加 大数据、人工智能、区块链、云计算等技术, 构建数 据驱动的证券公司运营及风险预警管控决策技术体 系。这就要求证券公司必须尽早尽快展开业务模式变 革、业务流程重构、客户服务优化及业务效率提升等 工作, 加速构建业务服务云平台和体系, 建立自身的 技术防火墙和风险监控网, 这也是未来一段时间内证 券公司急待推进的常态化工作内容。

\section{1. 证券行业金融科技生态应用} 点:

现阶段证券行业金融科技生态应用主要有以下三

1. 分布式技术: 金融基础设施转型升级、核心交 易平台、联合风控平台;

2.人工智能: 身份认证和用户画像、自动报告生 成、金融搜索引擎、智能投顾、智能客服、智能风控、 智能营销;

3. 区块链：金融资产发行与交易、清算结算、供 应链金融、数字货币。

证券公司数字化转型工作主要集中在两个方面: 一是在财富管理领域推出智能资产配置、场景化理 财、科技创新交易、智能助手等特色服务。二是在机 构服务领域打造智能交易、高速行情、量化投研、一 体化投资管理、数字虚拟员工等产品。数字化转型过 程中会衍生出金融风险与技术风险叠加形成的新型 体系化衍生风险，如何面向金融科技、证券行业产业 链和数字化转型深度融合, 打造一个即服务于客户及 公司运营、又能够进行特定用途以收集、评估、篮选 和聚合创新概念的创新和协同设计研发与验证平台, 以及智能风控子系统迫在眉睫。

这个平台总体上概括为前台、中台和后台三个体 系化功能关联子系统，用来对证券公司的市场资源、 信息资源、客户资源、技术资源，以及自身储备的知 识库资源进行整合集成。前台是证券公司核心价值创
造流程所框入的服务线和产品线，是面向市场的通道 和网络。中台是证券公司的运营管理流程所框入的运 营支持线和内部监管流程所框入的风险监控线和资 源管理线, 这是市场需求识别和价值分配的通道和网 络。后台是证券公司的核心价值创造支持流程所框入 的 IT 信息技术、人工智能、区块链、云计算等金融 科技支持线和人力资源支持线，这是市场管理、客户 管理、知识管理、数据管理、信息融合、产品设计与 服务、财务核算、风险管理、合规分析、资源储备和 动态配置与监控的主要通道和网络。此外, 后台还包 括证券投资分析需要的价值网络分析模型和价值关 联图谱, 这是投资管控的专家决策支持知识库和模型 库的应用模型。

\section{2. 国内外研究现状}

目前国内外对于金融产业链的研究相对较少, 就 金融产业的研究而言, 现有文献主要集中于其结构、 布局、关联等方面，而极少对金融产业链整体进行概 念厘清与分析比较, 更无对于金融产业链的重要分支 一证券产业链进行过详细研究。在国内研究方面, 束 兰根(2010)通过分析股权投资业务发展环境，提出以 股权投资为核心的金融产业链发展模式[1]。刘以研、 王胜今(2013)提出信息技术的变革、客户需求的变革 和市场竞争及金融机构商业模式的变革是形成移动 金融产业链的驱动因素, 并基于此构建出移动金融产 业链模型[2]。杨艳琳、周丹(2015)选取股票、期货、 互联网金融三类金融产品进行具体的金融产业链构 建, 为基于产业链的金融产业理论研究提供概念性基 础[3]。在国外研究方面, Sidney（1960)、Rajan(1998)、 Corpataux (2009) 等人梳理了金融产业的绩效对经济 的影响 [4-6], Malmbeg（2001）、Choi(2003)、Jessie (2004) 等人从金融产业集聚方面进行了研究[7-9]。 但从整体上看, 基于证券行业的全产业链价值分析尚 缺乏理论支撑。因此, 创新、构建、整合适用于证券 行业的全产业链模型, 不仅可以补充对于金融产业链 的研究, 而且对于实现证券行业“提质增效”具有理论 指导作用。

在金融科技研究领域，国内外相关成果大多聚集 于金融科技对金融产业的影响因素，运作方法以及发 展模式方面。在国内的相关研究中, 周昌发(2011)从 理论角度阐述了金融科技的内涵与保障方式 [10]。叶 子荣、贾宪洲(2011)使用面板数据模型实证探讨了金 融支持对于自主创新的影响[11]。王宏起、徐玉莲 (2012) 通过对金融科技内涵的界定和科技创新与金 融科技的相互作用角度分析，揭示了科技创新与金融 科技的协同发展机理 [12]。国外研究方面, Berger(2003)、Schinckus(2008)、Agbolade (2011)等人 研究了以信息通信技术为代表的科技创新对金融体 系发展的影响[13-15]。Ang(2010)和 Vasilescu(2011) 等人对金融体系中银行、资本市场及风险投资发展对 科技创新的作用进行了相关研究[17]。现有研究表明, 数据和科技是金融科技改革传统金融业的两大主要 
支撑。而对于具体的金融科技应用于金融产业特别是 证券行业, 从而构建完善的证券产业链广义资产管理 创新平台等方面的研究缺乏相应的理论构想与理论 支持。

综上所述, 本文以证券行业作为切入点, 剖析数 字化转型过程中, 金融科技、金融产业链和数字化转 型的结合与实际应用。在此基础上试图通过证券行业 数据管理公司和创新研发实验室的构建与完善, 来解 决证券行业长期缺乏而又急需的对于其产业链广义 资产管理平台的实际问题, 并提出具体且全面的实施 步骤与决策参考, 实现金融科技与数字化转型深度融 合。

\section{3. 金融科技赋能数字化转型的内容}

数字化转型不是简单的IT系统升级, 而是“IT升级 +管理模式变革+业务模式创新”的过程。通过组建证 券行业数据管理公司和创新实验室作为切入点, 打造 证券行业人工智能与大数据领域集服务、管理、成果 和收益于一体的数据管理和创新研发平台, 是数字化 转型成功的基础和技术保障。

\section{1. 金融科技与数字化转型深度融合的切入 点}

组建证券行业数据管理公司和创新实验室作为推 动金融科技与数字化转型深度融合的切入点, 承担和 实现如下功能目标: 证券行业服务创新和产品设计与 开发、业务模式探究、技术储备与运用、创新与整合、 技术成果和趋势跟踪研究与验证、联合智能风控、构 建涵盖工具库和模型库及行业知识图谱库于一体的 行业数据库、构建实战人才培养与实训于一体的技术 研发、服务、转化和实训平台。项目建设要具备行业 性、长远性、领先性和现实性。因此其定位主要有以 下几点:

1.核心业务能力的发动机。核心业务能力的持续 保持与发展, 核心风险管理能力的完善与提升, 核心 产品盈利能力的发掘与保持, 核心技术能力的跟踪与 转化。

2.核心源数据资源的发现与验证。证券行业的核 心源数据资源, 特别是涉及投资和市场标的物的价值 源数据资源及其宝贵和难得, 需要一个环境和平台来 发现、检验和获取。把行业的数据资源作为公司的数 据资产进行管理和价值挖掘, 编制行业数据资产目录 和数据资产价值评估模型, 实现行业数据的价值和市 场价值。

3. 证券行业知识图谱价值链的构建和维护。证券 行业是覆盖社会资产价值网络的显性和隐性最为集 中和反应敏感的环节, 因而其价值链所关联的行业知 识图谱模型非常复杂和广泛。需要专门进行构建行业 知识图谱模型库和持续地完善, 这本身就是一个价值 平台。
4.创新人才的实训地。创新人才需要创新知识和创 新技术的培训和实操, 这就需要环境试错、知识体系 与创新能力自评及其改进和提升训练。把平台建成证 券行业的数字孪生平台, 对从事高端技术和管理的人 员进行业务场景实训。

5.潜在价值的探测器。业务转型与重构本质上是发 现满足客户和业务发展的新的机会、或者创造新的资 源整合机会、或者设计新的价值增长模式。平台以其 聚合的创新资源、积累的创新知识和成果、构建和逐 步完善的创新环境，采用信息、数据、资讯和知识大 融合技术，进行资产流动的潜在价值的探测，面向不 同层面的使用者满足他们的价值发现需求。

在具体实施过程中，首先对业务流程进行梳理， 提出适合公司业务拓展和占有先机的业务和领域方 向设计与平台服务能力规划, 目的是构建一个即服务 于客户，又能够以收集、评估、篮选和聚合创新概念 的创新和协同设计研发与验证平台，用来对公司的市 场资源、信息资源、客户资源、技术资源，以及自身 储备的知识库资源进行集成整合，搭建证券行业数据 治理与服务体系和数据服务平台。依托这个平台，围 绕业务条线、产品条线和技术条线，对证券行业产业 链及其运行环境数字化, 并开展全方位、全产业链价 值分析的创新研究和设计。具体如下:

1.在业务条线，收集、评估和梳理产业链广义资产 的形式、类型、价值、归属与状态，设计与资产关联 的网络和资本聚合节点与模式，定义属于公司的业务 板块和相关的业务流程, 制定业务形式重构与创新构 想概念及其原型方案。业务形式重构与创新专家依托 原型方案互动进行业务条线的重新定义, 并开展资产 管理价值链中的价值贡献分析、合规与风险评估，形 成并提交可实施的业务形式重构与创新主题。

2.在产品条线, 收集市场需求和客户要求，同时对 公司的现存产品形态按照市场、价值、成本、时间、 粘性、风控、增长和品牌等多维度视角, 进行大数据 测量和全生命周期量化分析，结合公司运营战略和产 品研发战略目标, 聚合成公司的新产品概念及其关联 的业务条线和多维度监测, 及大数据服务监管规约与 大数据服务评价体系。然后与产品开发设计专家团队 互动进行产品属性定义、产品功能定义、市场定位和 服务模式设计，并进行产品的价值分析、风险分析与 先期服务及风险管理流程设计, 形成并提交产品创新 主题及其配套大数据服务原型, 实现对产品形态重 构。

3.在技术条线，收集业务信息、客户信息、运营信 息和核心价值创造流程信息等大数据资源, 应用大数 据处理技术聚合提炼出技术服务需求，构建业务、产 品、客户知识图谱库。以证券行业核心源数据资源的 聚合技术体系、证券行业核心知识图谱库构建模型, 及其证券行业大数据价值发现技术体系为核心，重构 公司技术条线。用重构产业实用技术创新原型与技术 创新专家团队互动, 确定和评估技术先进性、成熟性、 
体系化和行业竞争力, 测算技术创新的投入产出, 形 成并提交技术创新主题, 实现基于行业知识图谱价值 集合的技术创新。

通过搭建整合证券行业数据资产的收集、集成服 务专业化平台, 以自主知识产权推动证券行业的产业 链完善和价值链升级, 促进公司创新生态圈的打造和 资源化聚合与商业化培育, 培育大安全、大质量、大 智造、大数据的创新生态, 构建一个数字化、智能化 协同创新规则系统和标准规范体系及其商业化管理 模式，最后成果落实于共性技术支撑云服务平台。平 台将融合大数据、工业互联网、数据挖掘、质量数据 治理、知识管理、深度学习、知识图谱等技术, 为证 券行业的产业化协同创新提供共性技术的模型与工 具云化的服务支撑, 并与现行的线下运营平台对接和 互联互通。

\section{2. 组建证券行业数据管理公司}

组建数据管理公司是数字化转型落地的首要环 节, 也是金融科技与数字化转型深度融合的切入点。 要站在整个行业的高度从证券行业全产业链的视角 进行规划、设计和平台开发。要系统性的把握产业价 值链模型、实体产业资产证券化及其流动模式、监管 与风险控制模式等需要的流程化、定量化、指标化、 工具化、模型化、测量性，及其相关的技术开发、整 合、集成与应用研究, 规划和建设相应的数据管理平 台及体系, 构建证券公司产业链需要的工具库、模型 库、知识图谱库和全产业链价值网络数据库。数据管 理公司应涵盖以下子体系:

\subsection{1. 证券公司创新流程及其评价体系}

这是证券公司自身运作管控的范畴, 但是直接要 对接和激活创新研发实验室的全部项目管理与风险 管理流程。因此, 就管理体系及其支撑技术视角, 是 一个关键技术内容。这就需要构建流程化管理、过程 化测量监控体系和评价模型的设计与实现技术。

\subsection{2. 证券公司全产业链价值网络模型构建 体系}

这是证券公司在给自身定位、确定发展模式与价 值、利润实现所必须清晰的管控模式。证券公司全产 业链是从公司业务出发对所涉及的供应链和客户的 一种梳理、建模与量化的过程, 也是对自身内部业务 运作的一种审视和自查。需要管理和管理量化技术支 持。通过全产业链价值网络模型构建, 可以设计全产 业链价值网络数据库和产业链价值模型库。

\subsection{3. 实体产业资产证券化及其定价模型体} 系

这是证券公司最基础的技术基本功和核心竞争能
力。因为实体产业的发展和扩张是用其资产的规模来 体现的，而资产及其权益可以用股权回报来体现，这 种权益进行交易就可以进行定价。所以，不同的公司 在其自身的产业价值链资源的整合过程中, 会对同一 产业资产给出定价, 但是这种定价是不能保证相等 的, 即使在同一模式下进行证券化。由于这种差异, 使得不同的公司采取有差异的经营策略和资产管理 模式。

\subsection{4. 实体产业资产价值流动网络模型的构建 与流动价格测算体系}

这是控制资产管理收益风险和经营风险的一个基 础技术模型系统。证券公司要对其聚合的资本所能够 对接的实体产业资产价值流动网络进行网络化模型 的设计, 并依据模型对资产流动价格进行运筹, 取得 资本一资产对接过程的流动收益最大化。

\subsection{5. 资本伴随资产价值流动性风险控制与评 估模型体系}

这是控制经营决策风险和资产管理操作风险的一 个基础技术模型系统。证券公司要对其资产管理的操 作过程进行量化风险控制需要明确资产价值流动性 及其资本转换风险评估, 并对过程关键环节的资本风 险控制和资产价值流动与转换进行测量和评估。依据 这一模型系统, 实现操作风险监测与评估。

\subsection{6. 证券产业链业务与服务操作风险预估模 型及其管控体系}

这是证券公司控制业务和服务流程操作风险的一 个基础管理量化模型技术系统。

\subsection{7. 创新创意聚合技术体系}

这是证券公司把创新融入公司日常业务和经营活 动的一个市场需求、客户信息、流程信息、经营信息 的闭环逼近的创新创意收集与提炼技术。

\subsection{8. 创新项目管控与价值评价技术体系}

这一技术用于证券公司对较大投入的创新项目进 行风险管控和过程管理的量化管理和价值（财务收益 和潜在市场及其客户财务贡献价值）评估技术。

\subsection{9. 创新过程风险评估与控制技术体系}

这一技术的内涵和作用同上一技术。更强调对创 新项目的过程关键节点的风险传递和后续风险控制。

\subsubsection{0. 创新成果实用型转化技术体系}

这是对已有和已完成尚未使用的创新成果进行实 
用性评价和试用方法的评估。涉及知识产权引入与商 业技术壁垒的突破技术。

\subsubsection{1. 创新知识产权保护与服务技术体系}

这是证券公司创新常规的内容。

\subsubsection{2. 创新技能培训与实操环境构建技术体 系}

创新技能培训是创新人才持续的实践和实操过 程。一般传统的模式是公司用自身的业务去让创新人 才试错, 会增大创新成本、延长创新周期, 降低创新 成果的功效。因此需要通过创新研发实验室向创新人 才的创新能力提升与知识体系的学习与完善提供检 验、自测、评价、培训和操作环境。

\subsubsection{3. 证券行业数据、实体产业数据、相关} 资讯聚合和质量控制技术体系

数据与资讯聚合和融合技术是当前制约证券公司 价值发现、资产管理和经营运作风险控制的瓶颈技 术。而制约该技术发挥效用的是数据与资讯聚合和融 合的输入一既数据与资讯的质量。这里的质量控制技 术是指数据与资讯的质量控制、及数据与资讯聚合和 融合的质量控制技术。

\subsubsection{4. 资讯和数据融合与知识提取和编制技 术体系}

以上述全部成果进行技术集成, 构建证券行业知 识图谱价值链模型, 并搭建证券行业知识图谱库、全 产业链价值网络数据库和产业链价值模型库及其技 术应用引擎。

\subsubsection{5. 人才知识体系和实操能力培训与训练 在线博栾与协同技术体系}

这是证券公司人才知识体系和实操能力培训与训 练常规的内容。

\section{3. 建设创新研发实验室}

建设创新实验室同样是数字化转型的技术保障, 与数据管理公司相辅相成。创新实验室应具备以下十 大研发功能和技术实现:

\subsection{1. 证券产业链与产业链资产的价值传递 与转换功能网络动态组合}

该功能是用于在证券公司业务模式与产品构想创 新研究过程中, 提供资产产业化属性、资产流动性属 性、资产价值属性等与证券产业链激活资产的资本流 动性属性之间的价值网络关联关系; 资产与资本之间
价值传递与转换关系; 以及构建证券业务模式与产业 资产价值实现模式之间的运行网络关系, 并作为创新 研发实验室的基础关系模型。用于广义资产管理业务 及其产品构想、设计、验证和试用。引入资产所有者 并视其为客户参与网络资产价值实现的操作体验, 以 评估证券公司创新的业务模式、服务产品和技术支撑 能否达到预期或超预期效果。

\subsection{2. 证券公司内部资源集成整合与共享}

该功能是用于证券公司内部客户资源、业务流程 资源、各种金融市场交易数据资源、行业研究与金融 信息资源、内控与合规监管资源、定价模型及其估值 模型等各种资源的集成、整合与共享，并提供各种接 入的交互界面。

\subsection{3. 证券公司创新概念收集与聚合}

该功能是用于证券公司客户信息收集，聚合成客 户对公司的服务需求和产品的性能指标与功能要求, 收集公司内部业务流程的技术支撑需求和产品开发 系统对技术支持及其产品工具化需求, 收集证券行业 信息, 聚合成证券行业业务发展趋势。通过聚合, 形 成证券公司业务与产品和技术创新概念, 并给出创新 概念定义和价值评估。

\subsection{4. 证券公司业务流程设计与模拟验证}

该功能是用于业务创新中业务流程的设计、优化 与模拟验证。要依托证券公司的资源整合与共享子系 统动态地构建和组配业务流程设计、优化、评价和验 证环境, 并实现客户参与的闭环、创新专家及其业务 团队和公司相关业务条线全员参与的闭环, 形成与客 户体验闭环。

\subsection{5. 证券公司产品设计与开发和模拟}

该功能是用于证券公司产品创新中产品开发阶段 的设计与设计验证; 产品推送和服务流程设计; 产品 市场收益预估和收益模拟验证; 产品风控模型与风控 流程设计; 以及产品组合与组合收益测算。产品创新 要与市场实际运行进行对接和模拟评估, 要有客户参 与并与客户体验进行闭环。

\subsection{6. 证券公司技术支持系统、产品与服务工 具设计开发及效果评价}

该功能是用于证券公司在业务设计技术、产品设 计技术、业务流程监测技术、操作风险监控技术、经 营风险测量与量化技术、资源（知识、数据等）聚合 技术、人员培训及实操训练与考评技术、创新项目实 施与创新阶段成果评价及过程风险控制技术、创新项 目市场价值财务评价和预估技术等技术方面的工具 聚合和应用环境的搭建。 


\subsection{7. 产业资产证券化及其价值流动网络和 定价}

该功能是证券公司开展泛资产管理时必须依托的 系统平台。在把证券产业链向实体产业链对接过程 中, 需要建立证券资本资源与产业资产对接的媒介和 互换模型, 这就是产业资产证券化及其价值流动网络 与证券资本流动网络的共同节点。因此，在证券公司 业务与产品服务上, 就要向资本和产业实体客户提供 定价服务和流动性网络流程及其价值测算模型, 以帮 助客户实现资产与资本聚合和资产价值变现的业务 与产品和技术服务。基于这样的理念进行创新, 是以 实现客户价值需求的互利服务创新, 而不是仅限于个 别理财产品的推送, 所以能够触摸到行业的制高点和 产业链全局。而且也能够达到证券资本推动和促进实 体产业资产增值与实体经济升级转型整合的催化剂、 倍增器和粘合剂的社会使命。

\subsection{8. 证券公司内部运作管控体系诊断与有 效性测评}

该功能主要是针对证券公司在创新之前需要对自 身的运作管控体系进行必要的诊断, 以避免运作管控 失效。运作管控失效会给证券公司带来决策输入性风 险、方案执行操作性风险、监控盲区, 最终体现在财 务风险上。通过对证券公司运作管控失效模式及其风 险损失的量化分析, 可以建立在风险阈值内的风险管 控策略, 并评估策略的有效性。

\subsection{9. 证券公司创新人才培训与实操训练}

该功能主要针对证券公司业务与产品开发及技术 创新人才比较短缺, 创新人才知识体系和技术能力的 培训和实操需要借助于创新研发实验室的资源和证 券行业的数字孪生环境才能实现, 仅仅书本上的知识 和短期的集中培训是难以承担创新任务和胜任创新 岗位要求的。该功能可以实现创新知识体系的可视化 交互式学习、训练、自评和能力测试, 在线或离线进 行协同训练, 泛资产管理能力体系自我学习与完善 等。

\subsubsection{0. 证券大数据知识图谱价值链管理}

该功能主要针对证券核心源数据进行发现和集 成, 从客户、产品、业务视角, 构建证券行业知识图 谱库和价值链模型, 构成证券行业的知识管理和知识 迭代积累平台。集成证券核心源数据集成技术体系、 证券行业知识图谱构建技术体系、价值链实现及其风 险管理技术体系, 搭建 (合建、租用) 证券行业大数 据服务云平台。

\section{4. 建设大数据风险管理服务平台及证券行业 知识图谱库}

作为数字化转型关键环节的大数据风险管理服务 平台建设需涵盖如下内容:

\section{1. 证券业务一风险知识图谱}

证券业务知识图谱，以证券业务实现过程的程序、 规则、方法、岗位等为实体对象, 建立关联关系及其 路径网络图, 构建图模型。然后, 对证券业务一风险 知识图模型分析可能的演化范式, 根据客户风险偏 好, 建立风险一收益管控策略集和方案集, 并按照随 机图规划和智能专家系统优选一种风险一收益管控 路径和策略。证券业务一风险知识图谱的应用场景如 下:

1.证券行业风险画像, 按照公开来源数据和商业合 作数据、以及其他合规手段获得的数据, 作为风险画 像源数据, 捕捉数据分布特征, 充分过滤和屏蔽数据 的噪声,借助知识图谱引擎, 从风险和收益的角度对业 务板块进行画像, 对其风险进行定性的量化分析并支 持可视化;

2.证券业务链风险画像, 按照证券业务链对证券公 司内部风险以及外部风险向内部传导风险进行大数 据风险态势分析和趋势推测, 并支持可视化;

3.客户风险画像, 以行业景气度、地域差异性、所 在公司信用风险、政策和客户收入一支出为大数据的 源数据支持, 对客户风险进行画像, 对其进行大数据 风险态势分析和趋势推测, 并支持可视化。

\section{2. 风险图网络的演化范式及监测与测量}

证券业务风险成本一风险收益网络图的变化及其 演变的模式, 通常被人们有意或无意的进行掩饰。这 样做无外乎把其演化的风险成本一风险收益的收支 路径进行改变, 客观上躲避监管和风控。因此, 通过 该子体系的数据资源和监测系统, 对风险成本一风险 收益网络图的演化过程进行监视测量, 用大数据技术 和工具分析风险成本一风险收益网络演化态势, 管控 证券业务风险成本一风险收益。

\section{3. 风险成本一风险收益知识图谱引擎}

当建立了风险成本一风险收益网络图谱和知识图 谱, 就需要运用开源、建模、查询、数据可视化、语 义分析等图谱工具支持业务人员搭建自己负责的业 务风险成本一风险收益网络知识图谱, 建立风险图谱 演化态势图和进行态势仿真与评价。要想做到上述事 项, 需要建立业务应用子系统的运行框架和模型, 也 就是风险成本一风险收益知识图谱引擎。 


\section{4. 风险成本一风险收益账簿}

支撑业务风险成本一业务风险收益网络知识图谱 的量化模型是风险成本一风险收益账簿, 这也是业务 风险成本一业务风险收益网络知识图谱的一个子集。 面向证券业务庞大的体系, 需要针对明确的业务条线 建立风险成本一风险收益账簿（包括账簿元数据字 典、元数据模型及建模规则等）。

\section{5. 风险成本一风险收益 (识别、测量、危害、 效益、控制) 知识图谱库}

风险成本一风险收益（识别、测量、危害、效益、 控制）知识图谱库是按照业务条线和应用进行集成, 需要对应着图谱, 设计业务和应用组成的知识图谱索 引矩阵。风险知识实体需要采用风险识别与危害程度 分析的方法来评估, 包括成本实体、收益实体。需要 一步步自行建设和完善, 也可通过对外合作项目、也 可结合与其他公司做数据分析和数据建模的工作中 提取自身空缺的部分。

\section{6. 证券业务合规知识图谱库}

证券业务是国家对证券行业的强监管业务, 必须 符合国家监管和法律法规要求。所以, 需要针对证券 公司的业务搭建证券业务合规知识图谱。

\section{7. 证券业务链知识图谱库}

证券业务链是对证券业务的多个过程的业务接续 和衔接, 因此存在风险成本一风险收益的传递与转 换, 构成业务实体的关联和关系。需要搭建逻辑化的 柔性模型, 在使用时, 由使用者按照业务过程的实际 发生场景进行搭建, 并由知识图谱建立风险成本一风 险收益测量和量化评估模型。

\section{8. 证券业务价值链知识图谱}

证券业务价值链是对证券业务链的风险成本一风 险收益账簿及其流向的路径网络化模型描述。一个确 定的证券业务链图谱会有对应的价值链网络模型作 为证券业务链风险成本一风险收益分析框架, 和风险 管控优化的目标函数。因此, 需要构建证券业务价值 链网络及其价值链网络分析和测量与量化评估的知 识图谱。

\section{9. 证券业务一价值图谱及其量化模式和算 法库}

证券公司的所有业务, 都以业务链网络和价值链 网络及相应的图谱模型进行同构化, 所以, 证券公司 业务数据, 就可以用这种多维度、多空间、跨时空的 知识图谱及风险态势分析范式, 建立基于证券业务大 数据的证券业务数据建模和风险态势融合框架模型。
对应的量化工具、测量模式、价值模型及其配套算法, 大数据建模、风险态势融合与风险管控策略方案，按 照该服务平台的架构要求, 搭建大数据建模工具库、 风险态势融合可视化应用引擎及智能风险管控专家 子系统。

\subsection{0. 大数据风险管理服务平台体系架构}

\subsection{1. 平台体系架构}

平台采用多总线结构化、分布式体系架构，分四 类总线:

1.源数据总线及其元数据模型和数据字典;

2.功能驱动总线 (工具、功能、应用模块/功能子 系统、图谱引擎、规则引擎、风险成本一风险收益账 簿引擎等）及其工具箱、图谱库、模型库、算法库、 规则库、风险成本一风险收益账簿模板库、智能专家 子系统库等;

3. 业务总线及其证券业务应用子系统、业务链及其 知识图谱配置/定制引擎子系统、风险成本一风险收益 账簿定制/配置子系统、业务链风险仿真与风险评估子 系统、业务链风险管控合规评价子系统、业务风险数 据建模与态势融合子系统等;

4.门户服务总线及其配置/定制的客户端子系统或 用户模块。

\subsection{2. 平台服务运行逻辑}

1.平台通过门户服务总线与客户交互, 门户服务总 线加载用户需求识别智能专家子系统, 识别客户需 求, 辅助配置客户应用模板, 推送平台服务的可视化 界面, 接入客户信息及所需服务;

2. 客户通过平台门户进入平台, 平台按照客户的服 务需求, 由业务服务集成专家系统提出业务服务模板 供客户配置或定制, 并保留客户日志, 客户数据加载 和业务应用服务流程引擎将驱动业务应用子系统;

3.业务应用服务流程引擎引导业务应用服务子系 统集成服务功能、开辟业务应用服务数据池, 提供数 据、模板、规则、业务智能型专家系统、模型、算法 等, 并进行模型和算法跑合;

4.数据准备与提供、回收、分发、处置和再集成;

5.服务评价与改进。

\subsection{3. 平台模式选择}

平台模式可以为网络化运营的云服务平台模式, 也可以为单一客户个性化配置业务应用服务子系统, 包括: 客户数据的导入、业务风险成本一风险收益账 簿的配置、业务链网络可视化配置、业务价值链网络 的可视化配置、业务风险仿真环境与评价规则配置、 


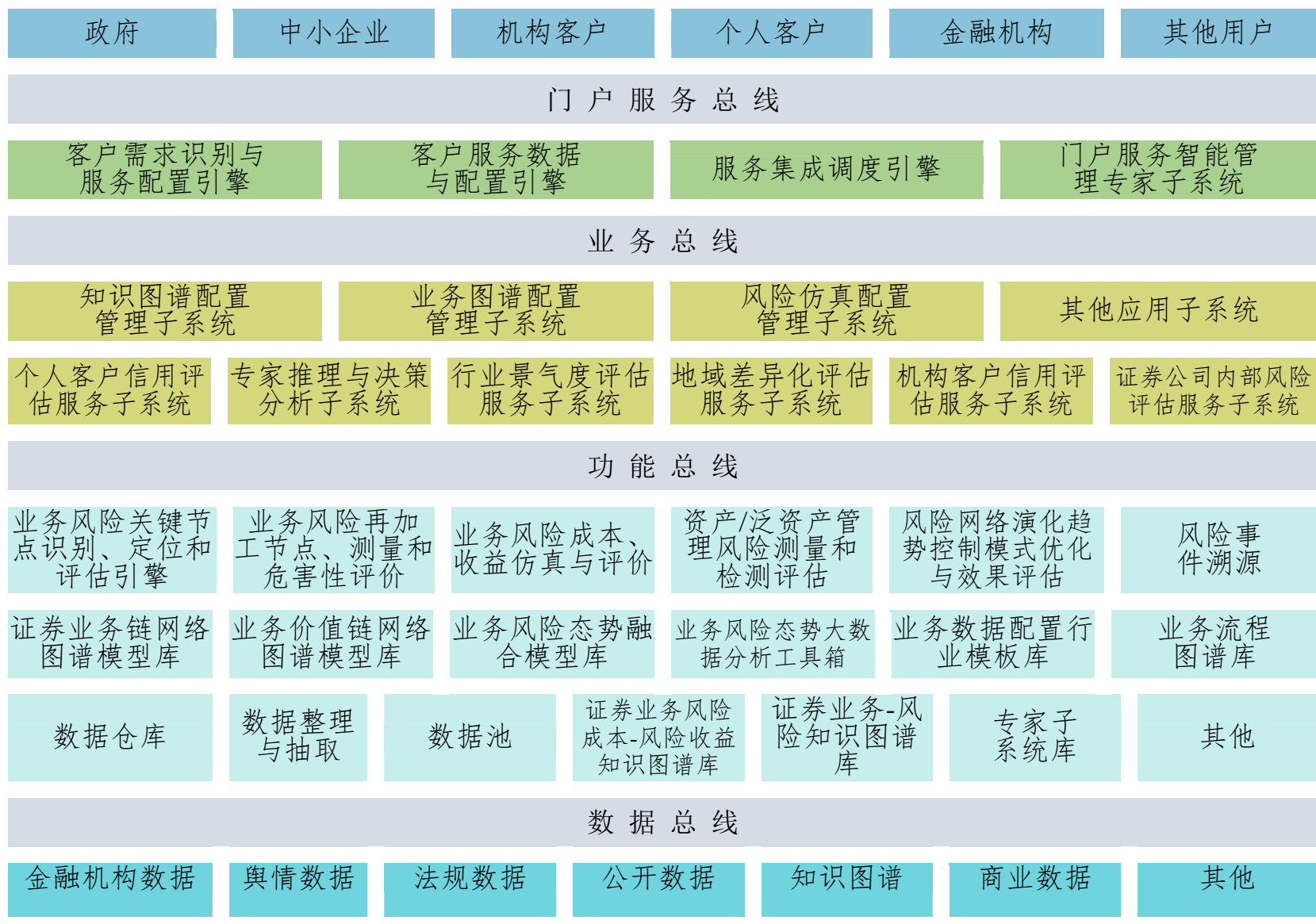

图 1 平台体系架构

\section{5. 建设证券大数据智能化服务专家系统}

无论是人工智能还是业务系统, 都需要专业化的 专家团队参与工作, 这就是专家决策支持系统的价值 和作用。

数字化转型进程中要建设证券大数据智能化服 务专家系统, 这是一个服务于证券公司各项业务的核 心纽带平台。大数据、人工智能技术的运用，很容易 产生衍生业务、衍生风险和新的商业模式, 如果在符 合合规风控要求的前提下进行设计开发, 可以充分发 挥业务服务及其业务衍生产品创新设计的想象空间。 因为服务创新和产品创新的前提是合规, 在此前提 下, 需要各系统对创新的价值进行评估、对创新风险 进行评价、创新产品和业务流程要进行跑合, 系统具 有多强的大数据及其人工智能算法应用生态共生空 间, 创新设计能力的想象空间就有多大。如果系统设 计一开始就面向具体的有限的业务, 互相割裂, 等于 把未来的发展空间在设计规划阶段就限制死了。

另外, 从技术支撑上, 要实现这个目标, 需要大 数据源的支持, 数据源体系及其整合和管理与应用数 据池的动态按需配置, 投资组合的规则设计, 投资标 的的资产价值评估模型，投资风险控制模型及其操作
风险管控专家模型等都要完备。

\section{6. 人工智能技术的应用和业务嵌入}

人工智能和大数据技术应用于证券公司的业务 有许多可为之处, 许多业务以及环节都可以介入人工 智能和大数据技术。但是在数字化转型和证券行业大 数据智能化服务云平台及其子系统建设推进和实施 过程中, 如果功利性太强, 急于求成, 基础性工作弱 化，尤其各子系统建设之间关联度不高，不能形成大 一统的体系, 就会导致嵌入人工智能失效, 或者在使 用人工智能技术时达不到预期效果。

人工智能已经发展到可以在算法、学习机理、体 系逻辑方面支持一些以信息融合、规则发现、趋势分 析、异常监测、优化运筹、知识积累、辅助决策等应 用。这就对建设证券行业大数据智能化服务云平台提 供了技术支撑。基于人工智能既可以重构传统业务、 衍生新业务、挖掘价值洼地、增强客户粘性, 也可以 提高合规监测和风险管理水平（见图 2)。

目前, 一些证券公司提出引入人工智能技术用来 提升和衍生自身业务能力及其产品开发。但是, 这些 尝试目前仍停留在仅对一些简单的算法做实验性的 
试算和结果比对。比如, 讲的比较多的采用人工智能 选股的方法框架, 只是把一些简单的算法按照选股准 则进行了应用试算, 试算的成果是回测, 这是不合理 的。我们知道，股市投资是以资金投入后的市场反应
为参考继续进行动态博峦的随机对策分析，而且几千 支交易的股票，会形成动态的价值挖掘网络，这是无 法采用场外事后回测的方法对算法及其运用准则和 组合逻辑进行评价的。这是由股票市场或其他交易型

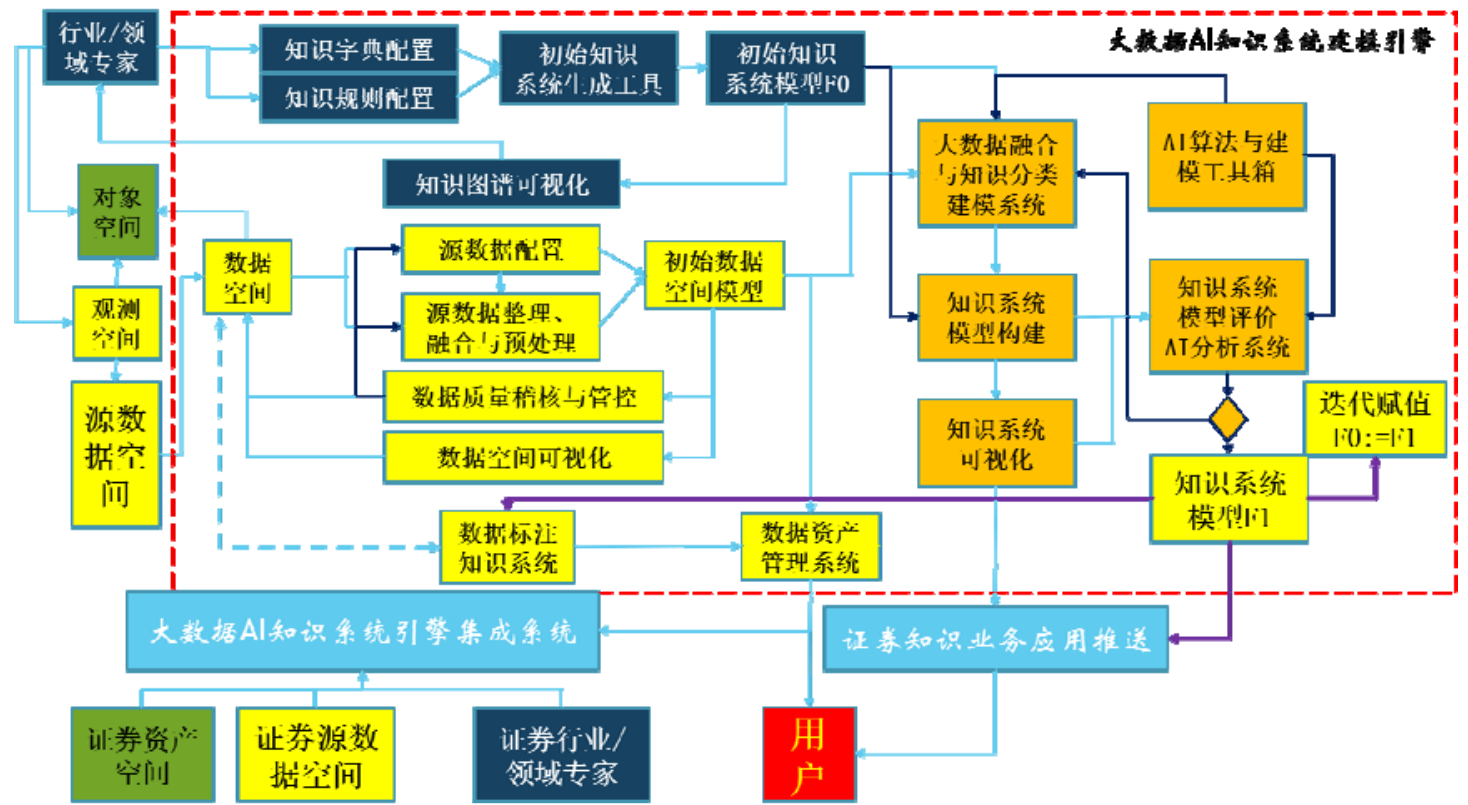

图 2 大数据 $\mathrm{AI}$ 知识系统建模引擎

市场的量子特性所决定的。正确的做法应该是采 用随机对策的量子算法进行投资组合及其交易策略 设计, 并依托大数据资源进行动态的博弯操作策略设 计与评价。所以, 证券公司在进行证券行业大数据智 能化服务云平台建设规划和业务板块搭建时, 需要充 分考虑应用场景的随机特性和业务人员、客户、监管 方、风险管理、合规等使用者的需求, 而不是任由第 三方 IT 公司或 IT 人员掌控, 大量的堆积设备和外购 软件产品、平台。需要公司的研发团队有针对性地根 据公司业务发展需要, 研发建设满足公司业务与客户 需求的具有自主知识产权的应用工具库、准则库、业 务规则库、业务知识库、专家模型库、法律法规库, 以及支撑上述的源数据集成管理系统。

\section{7. 结束语}

随着金融科技大潮的持续推进，大数据、人工智 能、区块链、知识图谱、云计算、物联网等新技术应 用与证券行业场景结合探索已经呈现了极高的热度, 金融科技手段与证券行业深度融合，助力证券公司转 型与业务重构已成为行业发展的必由之路。在此基础 上, 借助金融科技和数字化转型的深度融合, 推动证 券公司科学、规范发展, 即能有效减少监管压力, 提 高监管效能, 也能促进证券公司做大做强, 抵御国际 资本竞争, 助力证券市场向更深层次的发展迈进。

\section{REFERENCES}

[1] S. Langen, Research on the development of financial industry chain with equity investment as the core, New Financ. 9 (2010) 11-14.

[2] L. Yiyan, W. Shengjin, Mobile financial industry chain based on the context of information technology, Inf. Sci. 5 (2013) 138-141.

[3] Y. Yanlin, Z. Dan, On the construction and improvement of financial industry chain, J. Financ. Econ. 4 (2015) 14-17.

[4] S. Cottle, The Earnings Performance of the Consumer Finance Industry, J. Finance. 15 (1960) 387-406.

[5] R.G. Rajan, L. Zingales, Financial systems, industrial structure, and growth, Oxford Rev. Econ. Policy. 17 (2001) 467-482.

[6] J. Corpataux, O. Crevoisier, T. Theurillat, The expansion of the finance industry and its impact on the economy: a territorial approach based on Swiss pension funds, Econ. Geogr. 85 (2009) 313-334.

[7] A. Malmberg, P. Maskell, The elusive concept of localization economies: towards a knowledge-based theory of spatial clustering, Environ. Plan. A Econ. Sp. 34 (2002) 429-449. 
[8] S.-R. Choi, D. Park, A.E. Tschoegl, Banks and the world's major banking centers, 2000, Rev. World Econ. 139 (2003) 550-568.

[9] J.P.H. Poon, B. Eldredge, D. Yeung, Rank size distribution of international financial centers, Int. Reg. Sci. Rev. 27 (2004) 411-430.

[10] Z. Changfa, Protection mechanism for sci-tech finance development, China Soft Sci. 3 (2011) 72-81.

[11] Y. Ziirong, J. Xianzhou, Does financial support promote China's independent innovation?, Financ. Econ. 3 (2011) 10-18.

[12] W. Hongqi, X. Yulian, A measure model for synergy degree between sci-tech innovation and sci -tech finance and its application, China Soft Sci. 6 (2012) 129-138.

[13] A.N. Berger, The economic effects of technological progress: Evidence from the banking industry, J. Money, Credit Bank. (2003) 141-176.

[14] C. Schinckus, The financial simulacrum: The consequences of the symbolization and the computerization of the financial market, J. Socio. Econ. 37 (2008) 1076-1089.

[15] O.K.Agbolade, Information and communication technology and banksprofitability Iin Nigeria, Aust. J. Bus. Manag. Res. 1 (2011) 102.

[16] J.B. Ang, Research, technological change and financial liberalization in South Korea, J. Macroecon. 32 (2010) 457-468.

[17] L.G. Vasilescu, A. Popa, Venture Capital Funding-Path To Growth And Innovation For Firms, Ann. Ser. 1 (2011) 204-213. 